\title{
Expression of miR-15b, miR-29b, miR-219 and miR-222 microRNAs in rats with focal cerebral ischemia submitted to physical exercise
}

\author{
M.L.A. Cirino, L.B. Porsani, F.S. Lizarte Neto, M.F.G.S. Tazima, \\ R.F. Zimak, C.G. Carlotti Jr, B.O. Colli, L.F. Tirapelli and \\ D.P.C. Tirapelli
}

Departamento de Cirurgia e Anatomia, Faculdade de Medicina de Ribeirão Preto, Universidade de São Paulo, Ribeirão Preto, SP, Brasil

Corresponding author: D.P.C. Tirapelli

E-mail: lab.biomol.cirurgia@fmrp.usp.br

Genet. Mol. Res. 18 (4): gmr18213

Received November 19, 2019

Accepted October 16, 2019

Published November 30, 2019

DOI http://dx.doi.org/10.4238/gmr18213

\begin{abstract}
Cerebral ischemia is one of the main causes of death in Brazil, according to a survey by the Brazilian Society of Neurology in 2000, being the third cause of death after cardiovascular diseases and cancer; it is also one of the major causes of permanent sequels that can result in disability. In the last decades, experimental studies have shown beneficial effects of physical exercise associated with cerebral ischemia. Several molecular mechanisms are involved in the pathophysiology of cerebral ischemia, including changes in neurotransmitter expression profiles. Current research has highlighted the role of microRNAs both in the process of cerebral ischemia and in the regulation of neurotransmitters. Therefore, analyzing the expression of neurotransmitters and microRNAs associated with cerebral ischemia, as well as the role of the benefits promoted by physical exercise may contribute to the elucidation of possible molecular pathways with neuroprotective effect. Forty-eight rats were divided into four experimental groups: control, cerebral ischemia through middle cerebral artery occlusion, physical exercise and physical exercise associated with cerebral ischemia. Real-time PCR methodology was used to analyze miRNA expression of miR15b, miR-29b, miR-219
\end{abstract}


and miR-222. We did not observe significant differences in miRNA expression in brain tissue in rats submitted to cerebral ischemia, physical exercise and both treatments when compared with the control group. However, miR-222 expression increased in the cerebral ischemia group submitted to physical exercise, which may help promote cerebrovascular regeneration.

Key words: Brain ischemia; Physical exercise; microRNA; Neurotransmitter

\section{INTRODUCTION}

Stroke is the third largest cause of death in the West and patients with this disease have high rates of permanent disability compared to other diseases. Most strokes are ischemic and cause injury to a specific region of the brain by restriction of blood flow (Dhiraj et al., 2013). The incidence of stroke in the United States affects between 500,000 and 750,000 people annually, especially those over 65 years old (Kim et al., 2010). There are several important risk factors that contribute to cerebral ischemia, among them are hypertension, smoking, sedentary lifestyle and obesity (Bronner et al., 1995).

Ischemic stroke is a disease that is difficult to treat because it can occur both as a primary disease and secondary to systemic diseases (Nakano et al., 2002). The most common cause of ischemia is sudden occlusion of a blood vessel, resulting in an almost immediate loss of oxygen and glucose in the brain tissue. Absence of these factors can lead to several events, such as excitotoxicity, oxidative stress and inflammation, causing irreversible neuronal damage (Jablonska and Lukomska, 2011).

NMDA and GABAA neurotransmitter receptors control many essential brain functions and changes in their expression profile cause many disorders, such as memory dysfunctions. However, the molecular events involved in changes in GABA receptor expression remain unknown, one of the hypotheses being that these alterations are controlled by a new class of RNA molecule regulators called microRNAs. MicroRNAs (miRNAs) are involved in neuronal functions and several studies have shown that miRNAs modulate NMDA and GABAA neurotransmitter receptors. Many miRNAs participate in the regulation of signaling pathways of these neurotransmitter receptors (Mellios et al., 2009; Zheng et al., 2016).

MicroRNAs (miRNAs) are a newly discovered class of small RNA molecules containing 18-25 nucleotides that negatively regulate gene expression at the posttranscriptional level. The discovery of these small non-coding molecules favored our understanding of the mechanism of regulation of gene expression (Conti et al., 2009).

During focal cerebral ischemia, temporal changes occur in the expression profile of miRNAs throughout the brain. Bioinformatics analysis demonstrated that miRNAs regulated in cerebral ischemia target genes previously described altered after stroke in other studies (Dharap et al., 2009). Jeyaseelan et al. (2008) analyzed the ischemic tissue and found 56 miRNAs with altered expression levels in both reperfusion periods $(48 \mathrm{~h} / 24 \mathrm{~h})$, as well as 32 miRNAs were differentially expressed with $24 \mathrm{~h}$ of reperfusion and also 8 miRNAs exclusively in the period of $48 \mathrm{~h}$ of reperfusion. MicroRNAs are involved with neuronal functions and several studies have shown that miRNAs modulate GABA 
receptors. Many miRNAs participate in the regulation of signaling pathways of these neurotransmitter receptors (Mellios et al., 2008; Hen et al., 2011).

Motorized racing has been demonstrated as neuroprotective in several experimental models of ischemia. In addition to the increased neurogenicity in the jagged gyrus of normal rats, the race wheel increases the expression of proteins involved in synaptic trafficking, neurotrophin and neurotransmitter signal transduction pathways (Ploughman et al., 2005).

There are several epidemiological studies demonstrating that regular physical exercise is able to positively benefit health, increasing quality of life and decreasing the incidence of quality of life related dysfunction (Radák et al., 2006), as well as reducing risks and incidence of stroke (Ding et al., 2004). There are also studies that have shown that the sedentary lifestyle is related to the increased risk of strokes (Gillum and Ingram, 1996). During the last decades, experimental studies have demonstrated beneficial effects of physical exercise on cerebral ischemia, including increased survival, reduction of neurological deficit and maintenance of neurovascular integrity (Stummer et al., 1995). Some studies have demonstrated that physical exercise after $24 \mathrm{~h}$ to focal cerebral ischemia significantly improves neurological recovery and reduces the volume of infarction (Yang et al., 2002).

In the last decades miRNAs have been highlighted as potential brain biomarkers involved in many molecular mechanisms such as proliferation, differentiation, apoptosis, synaptic plasticity and memory. Several researches demonstrate that physical exercise can minimize the effects of traumatic brain injury as well as aging through the regulation of miRNAs. In this neuroprotective action of miRNAs, the association of the preservation of cognitive functions could be observed, with some miRNAs presenting increased levels of expression, for example miRNAs: miR-21, miR-34, 28a, miR-98a, miR -148b, miR-7a and miR-15b, and others with decreased expression levels: miR-105 and miR-133b (Fernandes et al., 2017).

Bao et al. (2014) performed experiments on mice submitted to physical exercise after traumatic brain injury. The acquisition of spatial learning and memory retention were evaluated. Next, microarray analyzes of miRNAs in the hippocampus were employed. The results demonstrated 32 differentially expressed miRNAs, 20 of which were hyperexpressed and 12 hypoexpressed. The authors also found that physical exercise may facilitate neuronal plasticity and improve cognitive function, since the results demonstrated that exercise recovered cognitive deficits in the hippocampus. The authors concluded that epigenetic mechanisms regulated by miRNAs may be involved in cognitive improvement induced by physical exercise.

There are specific miRNAs involved in the response of different types of physical exercise with an important role in the different types of muscle fibers and in the gene expression profile in both adults and the elderly population. In attention to health care involving physical exercise, there are increasing numbers of researches that highlight the role of miRNAs in the stimulation or inhibition of biological processes. Many miRNAs are essential mediators in the adaptations of the striated and skeletal striated musculature in both muscular hypertrophy and the regeneration process in adults and the elderly people. These miRNAs specifically involved with muscle fibers are called myomiRs (among them: miR-1, miR-133a, miR-133b, miR-206, miR-208, miR-208b, miR-486 and miR-499) also the process of myogeny (Ultimo et al., 2018). 
Little is known about the role of exercise in cerebrovascular diseases, especially in the process of cerebral ischemia and regulation of neurotransmitter receptors. Therefore, elucidating the role of microRNAs in the mechanisms involved in NMDA and GABAA receptor neurotransmitters imbalance with the benefits promoted by physical exercise in cerebral ischemia may contribute with new knowledge. Our hypothesis is that some miRNAs, which target receptors for NMDA and GABAA neurotransmitters, may be associated with the molecular pathways involved in exercise-associated cerebral ischemia. From an experimental model of cerebral ischemia by occlusion of the middle cerebral artery for $60 \mathrm{~min}$ followed by reperfusion of $24 \mathrm{~h}$ with physical pre-ischemic training, our objective was to evaluate miRNA expression: miR-15b, miR-29b, miR-219 and miR-222 in the tissue (ischemic focus).

\section{MATERIAL AND METHODS}

\section{General procedures}

The experiments were carried out according to the Ethical Principles for Experimental Animals (COBAO) and the study was approved by the Animal Experimentation Committee (CETEA) of the Medical School of Ribeirão Preto - University of São Paulo. Forty-eight adult male rats (Rattus norvegicus) weighing 280-310g were used. The animals career were randomly divided into four experimental groups: control (C): 12 animals sacrificed without being submitted to the surgical procedure; ischemic (I): 12 animals submitted to focal ischemia by middle cerebral artery occlusion (MCA) for 60 min followed by reperfusion for $24 \mathrm{~h}$, and then sacrificed; physical exercise (PE): 12 animals subjected to physical exercise; and, ischemic and physical exercise $(\mathrm{PE}+\mathrm{I}): 12$ animals subjected to the same treatment from group $\mathrm{PE}$ and focal cerebral ischemia for $60 \mathrm{~min}$ followed by reperfusion for $24 \mathrm{~h}$. Weekly measurements of the weight of the animals were held in the different study groups.

All animals were partially anesthetized by halothane inhalation and intubated with an orotracheal cannula. At two occasions during the ischemic period, arterial blood samples were collected for the determination of glycemia, $\mathrm{paCO}_{2}, \mathrm{paO}_{2}$ and $\mathrm{pH}$. MCA occlusion was carried out through the external carotid artery (ECA), which was ligated cranially and sectioned for the retrograde introduction of a $2.5 \mathrm{~cm}$ long obstructive 4-0 mononylon suture with one end thickened with silicone over an extension of $5 \mathrm{~mm}$. The suture was introduced until reaching the common carotid artery (CCA) and then cranially progressed through the internal carotid artery (ICA) to reach and obstruct the MCA.

After the period of ischemia, we proceeded to the removal of the obstructing thread, replacing the temporary clamp in the CCA to prevent the flow of blood and in the ICA to prevent the blood reflux. The proximal stump of the ACE was permanently connected and the temporary clamps were removed. Then, the skin and subcutaneous tissues were closed in animals from groups I and PE/I.

The animals went through a period of acclimatization for five days with velocities ( 5 to $18 \mathrm{~m} \mathrm{~min}$ ) and durations (5 to $15 \mathrm{~min}$ ), increasing progressively. The objective of the adaptation period was to reduce the stress levels presented during the manipulation and the use of the treadmill. The protocol was composed of a total period of 4 weeks. The session consisted of 2 $\mathrm{min}$ at a speed of $5 \mathrm{~m} / \mathrm{min}$ and this was gradually increased until reaching the speed of $18 \mathrm{~m} / \mathrm{min}$ in which the animals stayed for 30 min always with 0 degrees of inclination. When the animals showed any signs of fatigue or maladaptation to the use of the treadmill, the exercise was interrupted. During the training, no electric shocks were used on the animals, only slight touches 
with the hands to stimulate them. In a persistence situation, the animal was removed so that it could rest and recover. The training only resumed when the animal was fully recovered.

\section{Analysis of micrornas}

For the analysis of gene expression, all 12 animals were used per group. A fragment was obtained from each animal of the four groups.

Total RNA was extracted with Trizol reagent (Applied Biosystems, USA) according to the manufacturer's instructions. To verify the integrity of the RNA obtained, each sample was subjected to electrophoresis on agarose gel 1\% RNA and put through the spectrophotometer that provides the RNA concentration in a sample of 1 to $2 \mu \mathrm{L}$. In addition to the concentration, this device provides us with values of a reason relating to the integrity of the samples (260/280 ratio). The ideal range to be obtained is 1.7 to 1.9 .

To prepare the real-time polymerase chain reaction (PCR), reverse transcription of RNA samples was performed using the High-Capacity cDNA kit (Applied Biosystems, USA). The cDNA was amplified with quantitative Real Time Polymerase Chain Reaction (q-PCR) using TaqMan Master Mix (Applied Biosystems) for microRNAs reaction.

U6 was used as endogenous control for the reaction of the microRNA and the gene, respectively. All reactions were carried out in duplicate and analyzed with the 7500 Sequence Detection System apparatus (Applied Biosystems).

\section{Statistical analysis}

Data concerning the microRNA in the various groups were analyzed statistically by Kruskal-Wallis test followed by the Bonferroni post-test using the GraphPad Prism software (GraphPad Software, San Diego, CA, USA). The level of significance was set at $\mathrm{P}<0.05$ for two-tailed tests.

\section{RESULTS}

We did not observe significant differences in the expression of miRNAs, miR-29b, miR-219 and miR-222 in brain tissue of rats submitted to cerebral ischemia, physical exercise, or both when compared to the control group (Figures 1, 2, 3 and 4).

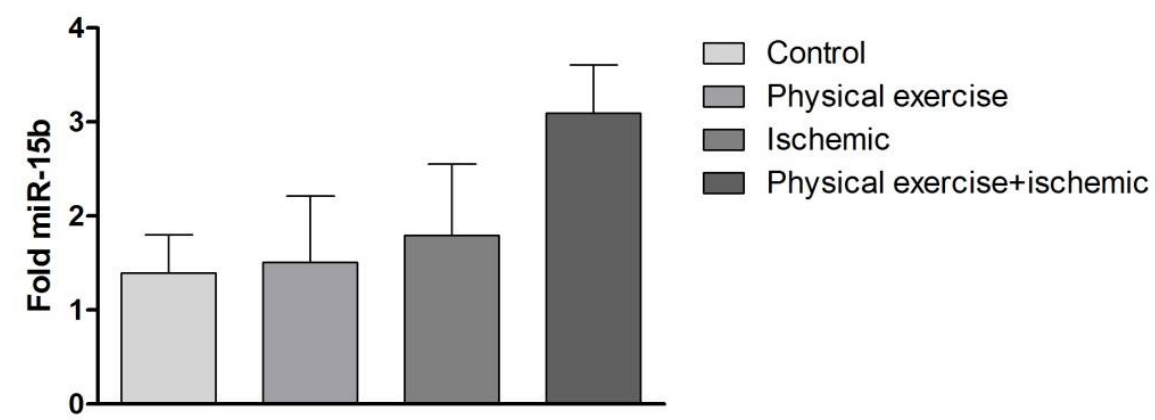

Figure 1. Mean values ( \pm standard error) of microRNA-15b expression in brain tissue between the treatment groups. 


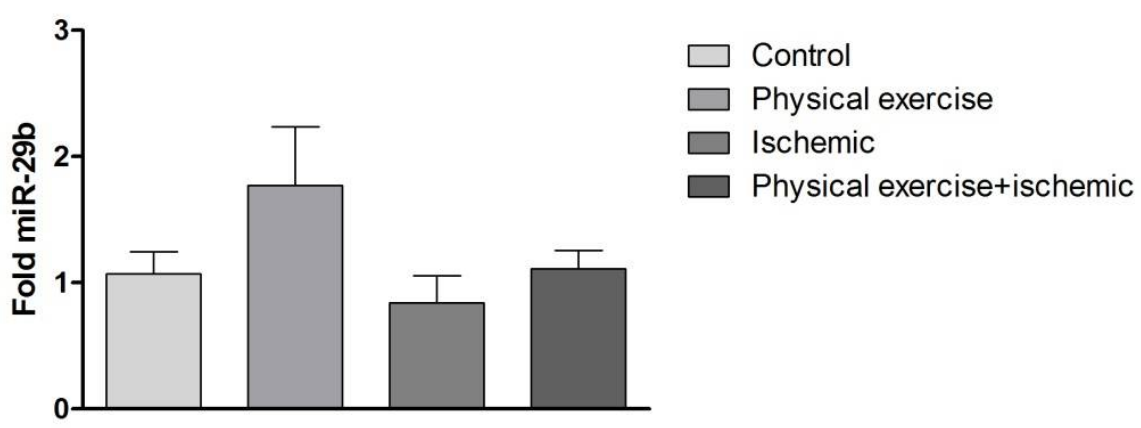

Figure 2. Mean values ( \pm standard error) of microRNA-29b expression in brain tissue between the treatment groups.

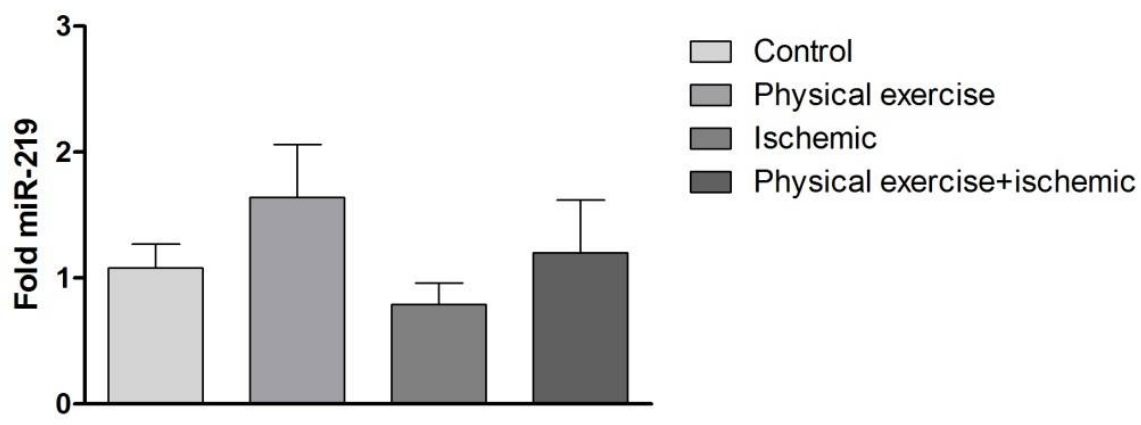

Figure 3. Mean values ( \pm standard error) of microRNA-219 expression in brain tissue between the treatment groups.

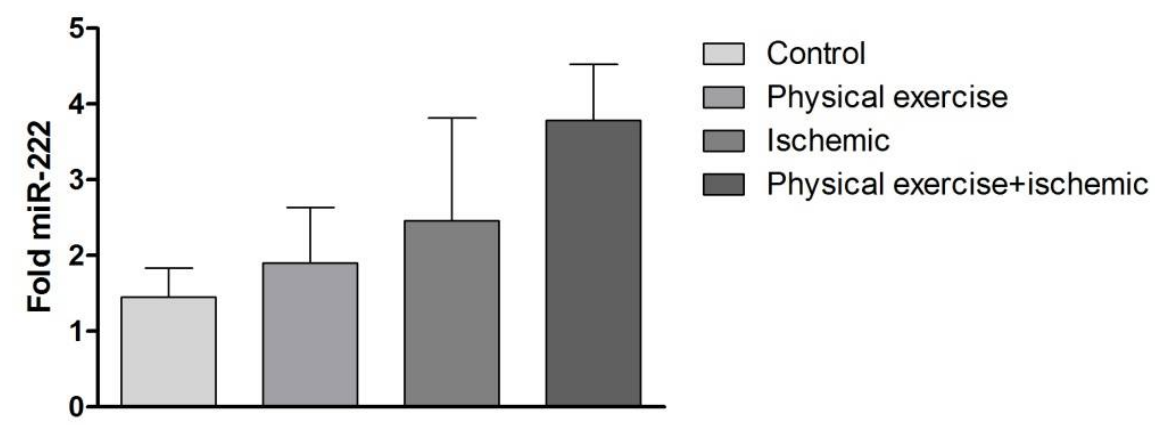

Figure 4. Mean values ( \pm standard error) of microRNA-222 expression in brain tissue between the treatment groups.

\section{DISCUSSION}

The practice of regular physical exercise is an important factor for the maintenance of a healthy life, since, evidences demonstrate its association with low risk of mortality and low incidence of more prevalent chronic diseases, such as: cardiovascular diseases, stroke, 
metabolic, type 2 diabetes and some types of cancer: prostate, colon, breast, etc. It is also possible to highlight the benefits of physical exercise in psychic diseases such as depression, for example (Fernández-Sanjurjo et al., 2018). This study aimed to evaluate the expression of some miRNAs previously identified as regulators of neurotransmitter receptors in animals submitted to exercise associated to focal cerebral ischemia.

Researchers point out the miRNAs as potential biomarkers for the different intensities of physical exercise training directly involved in various physiological processes and with different response depending on the intensity applied during the training (Schmitz et al., 2018).

The search for elucidation of which molecular pathways are associated with the neuroprotection provided by physical exercise during the cerebral vascular accident can effectively contribute to minimize the damage caused by this disease. In this context the miRNAs are highlighted as modulators of the expression of neurotransmitter receptors known to be involved in the ischemic process.

The miR-15 family (miR-15a and miR-15b) is involved in increased cell cycle gene expression, which inhibition in adult mice submitted to ischemia and reperfusion model promotes neuroprotection. Inhibition of miR-15 promotes increased expression levels of genes responsible for mitochondrial functions, cell survival and apoptosis blockade (Ooi et al., 2014).

Altintas et al. (2016), evaluated the expression profile of miRNAs, including miR15 , in rats submitted to a model of focal cerebral ischemia due to occlusion of the middle cerebral artery (MCAo) and diabetes (DM) induced by Streptozotocin (STZ). Ischemic preconditioning (IPreC) was performed for $72 \mathrm{~h}$ prior to transient MCAo to assess a neuroprotective effect against ischemia and reperfusion. The expression level of miR-15 was not altered in the DM and MCAo groups; however, hypoexpression of the same was observed in the IPreC group.

Physical exercise may improve functional recovery in spinal cord injury (SCI) and it has been suggested that some miRNAs are responsible for this process. In a study using rats submitted to exercise after SCI, a decrease in miR-15b expression levels was observed (Bhalala et al., 2013).

Our results support the conclusions of Altintas et al. (2016), since we also did not observe differences in miR-15b expression levels. The miR-29 family consists of three members (miR-29a, miR-29b and miR-29c). MiR-29a and miR-29b are present on chromosome 6 in mice and chromosome 7 in humans, while miR-29c on chromosome 1 in both species. MiR-29a and miR-29b are directly involved in the regulation of brain development and have high levels of expression in the brains of these animals as adults. The miR-29 family is predominantly more expressed in astrocytes when compared to neurons (Ouyang et al., 2014).

Bao et al. (2014) investigated the expression of miR-29b in neurodegeneration associated with acute ischemic stroke (CVA) by occluding the middle cerebral artery. It was observed that the increase of miR-29b expression levels had neuroprotective action and in contrast the inhibition of miR-29b potentiated neural cell death. The authors concluded that this paper provides the first evidence that loss of miR-29b in the ischemic focus is a key factor for stroke injury.

Experimental studies have demonstrated the participation of some miR-29 family members in swimming training-induced cardiac hypertrophy, thus being considered 
potential circulating biomarkers in the assessment of recovery in patients with hypertrophic cardiomyopathy. Swimming training re-establishes rno-miR-29ac cardiac levels, leading to improved ventricular function by preventing collagen deposition in infarcted hearts (Silva et al., 2017).

We did not observe differences in miR-29b expression levels in the rats, allowing us to suggest that the experimental design conditions, such as the duration of the ischemia period as well as the reperfusion time are directly associated with the standard expression of miR-29b. Schmitz et al. (2018) found increased expression levels of miRNAs miR-222 and miR-29c induced by high-intensity interval training (HIIT) in adult individuals in both men and women. These authors associated both miRNAs as potential cardiac biomarkers in response to HIIT with significant differences evidenced in the study between rest and physical exercise.

Liu et al. (2015) observed an increase in the expression levels of miR-222 in the blood after exercise with a stationary bicycle and described that physical exercise provides cardiac protection as a promising tool for regenerative therapies. However, little is known about the cardiac phenotypes involved in this process and suggests that further studies are needed to understand the role of miRNAs in the regeneration of cardiomyocytes, since they may contribute to important clinical implications.

Although we did not observe a statistically significant difference in our study, miR222 presented increased levels of expression in animals submitted to cerebral ischemia associated with physical exercise. Therefore, our results corroborated both with Schmitz et al. (2018) and with Liu et al. (2015). Both studies demonstrated the protective role of physical exercise with increased expression of miR-222 in the cardiovascular system that leads us to suggest a possible systemic vascular action of this miRNA also targeting cerebrovascular regeneration.

The miR-219 is a specific brain miRNA and negatively regulates the expression of the NMDA neurotransmitter receptors, being involved with several diseases of the Nervous System and has diminished levels of expression in experimental model of epilepsy as well as cerebrospinal fluid of epileptic patients (Zheng et al., 2016).

In a previous study by our group (unpublished), of an experimental cerebral ischemia model by occlusion of the middle cerebral artery for $90 \mathrm{~min}$, followed by reperfusion of $48 \mathrm{~h}$, associated or not with experimental chronic alcoholism, miRNA-219 expression was evaluated in the blood and in animal tissue by the time PCR technique. We also analyzed the gene and protein expression of the NMDA receptor in the tissue of the ischemic focus of these animals. The expression of miR-219 in brain tissue was lower in the groups submitted to cerebral ischemia, alcoholism and in the association of the two groups when compared to the control group. However, in the blood, the expression of miR-219 was lower in the groups submitted to alcoholism and in the group where alcoholism was associated with cerebral ischemia when compared to the control group. It was also observed that the gene and protein expression of the NMDA receptor in brain tissue was higher in the groups submitted to cerebral ischemia, alcoholism and in the association of the two groups when compared to the control group. Possible modulation of NMDA receptor NR1 by miR219 was observed, with an inverse correlation between expression of NMDA receptor NR1 and miR-219.

We did not find differences in miR-219 expression levels in the various treatment groups. Because it is a specific miRNA of brain tissue, competition between miRNA and 
antagonistic actions may occur. Further studies are needed to elucidate the interaction between the pathophysiology of cerebral ischemia associated with exercise. Other miRNAs should be evaluated and with different durations of cerebral ischemia as well as different reperfusion periods.

We did not observe significant differences in the expression of the miRNAs miR$15 \mathrm{~b}, \mathrm{miR}-29 \mathrm{~b}, \mathrm{miR}-219$ and miR-222 in the brain tissue in the groups submitted to cerebral ischemia, physical exercise and in the association of the two groups when compared to the group control. However, miR-222 increased expression levels in the exercise group associated with cerebral ischemia, which may be associated with cerebrovascular regeneration.

Research has proven the important role of miRNAs in the pathophysiology of the post-ischemic process, where they may have possible therapeutic potential by regulating their targets and promoting recovery and repair of brain tissue, activating mechanisms such as angiogenesis and neurogenesis, promoting a neuroprotective effect (Khoshnam et al., 2017). Therefore, further studies that aim to elucidate the therapeutic potential of miRNAs both in injuries caused by cerebral ischemia as well as the neuroprotection provided by physical exercise are warranted.

\section{CONFLICTS OF INTEREST}

The authors declare no conflict of interest.

\section{REFERENCES}

Altintas O, Ozgen Altintas M, Kumas M and Asil T (2016). Neuroprotective effect of ischemic preconditioning via modulating the expression of cerebral miRNAs against transient cerebral ischemia in diabetic rats. Neurol. Res. 38(11): 1003-1011.

Bao TH, Miao W, Han JH, Yin M, et al. (2014). Spontaneous running wheel improves cognitive functions of mouse associated with miRNA expressional alteration in hippocampus following traumatic brain injury. J. Mol. Neurosci. 54 (4): $622-629$.

Bhalala OG, Srikanth M and Kessler JA (2013). The emerging roles of microRNAs in CNS injuries. Nat. Rev. Neurol. 9(6): 328-339.

Bronner LL, Kanter DS and Manson JE (1995). Primary prevention of stroke. J. Med. 33: 1392-1400.

Conti A, Aguennouz M, La Torre D, Tomasello C, et al. (2009). miR-21 and 221 upregulation and miR-181b downregulation in human grade II-IV astrocytic tumors. J. Neurooncol. 93: 325-332.

Ding YH, Young CN, Luan X, Li J, et al. (2005). Exercise preconditioning ameliorates inflammatory injury in ischemic rats during reperfusion. Acta Neuropathol. 109: 237-246.

Dharap A, Bowen K, Place R, Li LC, et al. (2009). Transient focal ischemia induces extensive temporal changes in rat cerebral microRNAome. J. Cereb. Blood Flow Metab. 29(4): 675-687.

Dhiraj DK, Crysanthou E, Malluci GR and Bushell M (2013). miRNAs-19b, -29b-2* and -339-5p show an early and sustained up-regulation in ischemic models of stroke. Plos One. 8: e83717.

Fernandes J, Arida RM and Gomez-Pinilla F (2017). Physical exercise as an epigenetic modulator of brain plasticity and cognition. Neurosci. Biobehav. Rev. 80: 443-456.

Fernández-Sanjurjo M, de Gonzalo-Calvo D, Fernández-García D, et al, (2018). Circulating microRNA as Emerging Biomarkers of Exercise. Exerc. Sport Sci. Rev. 46(3):160-171.

Gillum RF and Ingram DD (1996). Relation between residence in southeast region of the United States and stroke incidence. The NHANES I Epidemiologic Followup Study. Am. J. Epidemiol. 144: 665-673.

Hen JF, Luo YM, Wan XH and Jiang D (2011). Biogenesis of MiRNA-195 and its role in biogenesis, the cell cycle, and apoptosis. J. Biochem. Mol. Toxicol. 25: 404-408.

Jablonska A and Lukomska B (2011). Stroke induced brain changes: implications for stem cell transplantation. Acta Neurobiol. Exp. 71: 74-85.

Jeyaseelan K, Lim KY and Armugam A (2008). MicroRNA Expression in the Blood and Brain of Rats Subjected to Transient Focal Ischemia by Middle Cerebral Artery Occlusion. Stroke. 39: 959-966. 
Khoshnam SE, Winlow W, Farbood Y, Moghaddam HF, et al. (2017). Emerging Roles of microRNAs in Ischemic Stroke: As Possible Therapeutic Agents. J. Stroke. 19(2): 166-187.

Kim Y, Leem JG, Sim JY, Jeong SM, et al. (2010). The effects of gabapentin pretreatment on brain injury induced by focal cerebral ischemia/reperfusion in the rat. Korean J. Anesthesiol. 58: 184-190.

Liu X , Xiao J, Zhu H, Wei X, et al. (2015). miR-222 is necessary for exercise-induced cardiac growth and protects against pathological cardiac remodeling. Cell Metab. 21(4): 584-95.

Mellios N, Huang HS, Baker SP, Galdzicka M, et al. (2009). Molecular Determinants of Dysregulated GABAergic Gene Expression in the Prefrontal Cortex of Subjects with Schizophrenia. Biol. Psychiatry. 65: 1006-1014.

Nakano H, Colli BO and Roselino SRJ (2002). Análise da respiração mitocondrial em tecido cerebral de gato após isquemia e reperfusão. Acta Cirúrgica Brasileira. 17.

Ooi JY, Bernardo BC and McMullen JR (2014). The therapeutic potential of miRNAs regulated in settings of physiological cardiac hypertrophy. Future Med. Chem. 6(2): 205-22.

Ouyang YB, Xu L, Yue S, Liu S, et al. (2014). Neuroprotection by astrocytes in brain ischemia: importance of microRNAs. Neurosci. Lett. 565: 53-58.

Radák Z, Toldy A, Szabo Z, Siamilis S, et al. (2006). The effects of training and detraining on memory, meurotrophins and oxidative stress markers in rat brain. Nerochem. Int. 49: 387-392.

Schmitz B, Rolfes F, Schelleckes K, Mewes M, et al. (2018). Longer Work/Rest Intervals During High-Intensity Interval Training (HIIT) Lead to Elevated Levels of miR-222 and miR-29c. Front Physiol. 17(9): 395.

Silva GJJ, Byea A, el Azzouzib H and Wisløff U (2017). MicroRNAs as Important Regulators of Exercise Adaptation. Prog. Cardiovasc. Dis. 60(1): 130-151.

Stummer W, Baethmann A, Murr R, Schurer L, et al. (1995). Reduced mortality and brain damage after locomotor activity in gerbil for brain ischemic. Stroke. 25: 1862-1869.

Ultimo S, Zauli G, Martelli AM, Vitale M, et al. (2018). Influence of physical exercise on microRNAs in skeletal muscle regeneration, aging and diseases. Oncotarget. 9(24): 17220-17237.

Yang YR, Wang RY and Wang PSG (2003). Early and late treadmill training after focal brain ischemia in rats. Neurosci. Lett. 339: 91-94.

Zheng H, Tang R, Yao Y, Ji Z, et al. (2016). MiR-219 Protects Against Seizure in the Kainic Acid Model of Epilepsy. Mol. Neurobiol. 53(1): 1-7. 